\title{
Les images des géographes : appropriation et restitution
}

Caroline ABELA

\section{Introduction}

C'est à partir de l'exemple d'archives de géographes que nous interrogeons la notion de responsabilité. Ces archives sont celles du centre d'information scientifique et technique "Regards » ${ }^{1}$, de l'unité de recherche "Passages », unité mixte du CNRS, des universités de Bordeaux et Bordeaux Montaigne, de l'université de Pau et des Pays de l'Adour et de l'École nationale supérieure de l'architecture et du paysage de Bordeaux (EnsapBx). Ce sont essentiellement des cartes et des photographies de terrain prises entre les années 1960 et 2000 dans des pays dits des «Suds » c'est-à-dire en Afrique, en Amérique latine, Asie et Océanie.

Elles ont fait l'objet de plusieurs projets d'archivage numérique depuis 2005 ; aujourd'hui ces derniers sont menés dans le cadre du consortium ImaGEO ${ }^{2}$, labellisé par la Très grande infrastructure de recherche (TGIR) Huma-Num du CNRS.

Nous mettrons l'accent sur deux types de responsabilités, en premier lieu celles liées au traitement documentaire et à la qualité des métadonnées qui sont produites. Celles-ci impliquent un travail réflexif et critique sur la constitution de représentations culturelles et sociales qui peuvent être archétypales. Et nous aborderons en deuxième lieu une responsabilité qui concerne l'accessibilité de ces archives, en particulier là où elles ont été originellement produites.

\footnotetext{
${ }^{1}$ http:/ / www.regards.cnrs.fr/

2 http://imageo.hypotheses.org/
} 
Ces deux responsabilités impliquent des ingénieurs documentalistes, des archivistes et des chercheurs. Elles sont sous-tendues par une dimension éthique qui se comprend par rapport au contexte spécifique dans lequel ces archives ont été produites.

\section{Contexte}

Les archives du centre d'information scientifique et technique (IST) « Regards » ont été constituées au cours de différentes périodes historiques de la discipline géographique, qui se sont succédé ou qui ont pu coexister en s'articulant les unes aux autres et qui reflètent une évolution des savoirs. Il s'agit de la géographie tropicale puis de la géographie du développement, ces deux périodes succédant à la géographie coloniale.

Cette histoire disciplinaire, et en particulier à Bordeaux, a fait l'objet de différentes publications, notamment dans la revue Les Cahiers d'Outre-Mer ${ }^{1}$. La géographie coloniale s'est appuyée, entre autres, sur la création de la Société de géographie commerciale de Bordeaux, en 1874. La géographie tropicale s'est développée en même temps que se développaient les relations avec l'OutreMer, c'est-à-dire avec l'Afrique, les Antilles et l'Amérique latine, à partir de 1945 et jusqu'au début des années 2000.

C'est en 1968 qu'est créé le centre de ressources «Regards », en même temps que le Centre d'études de géographie tropicale. Parmi les objets d'étude phare de cette géographie tropicale figurent des inventaires régionaux ou nationaux qui ont donné lieu à l'élaboration d'atlas. Le centre «Regards » est dépositaire d'une série d'atlas produits dans ce cadre, dont un sur l'île de La Réunion, paru en 1975, qui est le premier d'une collection «Atlas des départements français d'Outre-Mer». Cette collection comporte trois autres atlas, consacrés à la Martinique en 1977, à la Guyane en 1979 et à la Guadeloupe en 1980.

\footnotetext{
${ }^{1}$ Voir notamment :

MAIRE (Richard), "Un cas d'école : l'évolution de la géographie bordelaise depuis 1876 », Dynamiques environnementales, $\mathrm{n}^{\circ} 27,2011$, p. 11-37.

BOUQUET (Christian), Les géographes et le développement : Discours et actions, Pessac, Maison des Sciences de l'Homme d'Aquitaine, 2010, 287 p.

VELASCO-GRACIET (Hélène), Les tropiques des géographes, Pessac, Maison des Sciences de l'Homme d'Aquitaine, 2008, 235 p.
} 
Le centre acquiert par ailleurs un fonds important de cartes qui complètent ces atlas produits par le laboratoire. Il conserve également les photographies de terrain de géographes, qui sont surtout des diapositives. Ces dernières ont été collectées essentiellement dans les années 1970 et 1980 et reflètent les thématiques et les zones géographiques qui sont alors étudiées par les chercheurs.

Autour des années 1980 émerge la géographie du développement, dans la continuité de la géographie tropicale, avec des travaux orientés notamment vers la dynamique des milieux et des sociétés. Le centre d'IST «Regards » accroit alors ses collections de cartes et de photographies de terrain, accumulant peu à peu cinquante ans de patrimoine scientifique de la géographie. Ces collections représentent aujourd'hui 14000 cartes, 215 images satellitaires, 22000 photographies aériennes et 18000 diapositives. Cet héritage s'inscrit donc bien dans cette histoire disciplinaire et explique la volonté du centre de considérer ce contexte pour déterminer ses choix méthodologiques de traitement documentaire et de mise à disposition de ces fonds.

Ces questions méthodologiques et éthiques sont au cœur des travaux menés au centre « Regards » et au sein du consortium ImaGEO.

\section{Qu'est-ce qu'ImaGEO?}

ImaGEO répond à un besoin de la communauté scientifique, des géographes en particulier et plus largement des sciences humaines et sociales, d'accessibilité et de valorisation de données, qui sont surtout des images, en l'occurrence, des cartes et des photographies (diapositives, plaques de verre, etc.). C'est un réseau de partenaires qui se rassemblent autour d'objectifs opérationnels de mise à disposition de corpus, de nouveaux instruments de recherche. Ces partenaires mutualisent leurs moyens, leurs compétences, pour contribuer au développement et à l'évolution d'outils pour l'exploitation de ces corpus au service de la communauté scientifique et au-delà de la société civile. ImaGEO est à la fois un espace de réflexion et un espace de production qui a permis la mise à disposition de nouveaux matériaux de recherche, de nouveaux objets, lesquels offrent de nouvelles perspectives de recherche, par exemple des analyses diachroniques.

Une des réflexions menées concerne de «bonnes pratiques » éthiques quant au traitement et à la valorisation des données. Ces réflexions se sont développées en collaboration avec un groupe de travail Éthique et droit en sciences humaines et sociales spécialisé sur le sujet. 


\section{Ethique et droit en sciences humaines et sociales}

Ce projet, lancé en 2011 par Véronique Ginouvès et Pascal Garret, a donné lieu à un groupe de travail composé de chercheurs, documentalistes et juristes. Celui-ci a pour objectif de rédiger un guide de bonnes pratiques en termes éthiques et juridiques autour de la collecte de données, leur traitement, leur archivage, leur description, leur diffusion et leur réutilisation. La rédaction de ce guide prend la forme d'un carnet sur Hypothèse ${ }^{1}$ qui rend compte des travaux du groupe.

\section{Qualité des métadonnées}

De quelle manière documenter les collections du centre «Regards » aujourd'hui ? Nous prendrons l'exemple de photographies. Prises principalement dans les années 1970, 1980 et 1990 par les géographes sur leurs terrains, celles-ci ont fait l'objet d'annotations de leurs auteurs, et aussi de documentalistes qui se sont succédé dans les équipes. Elles ont ainsi été décrites au fur et à mesure de leur constitution avec les termes, les catégories conceptuelles et analytiques, d'une époque. Quelle lecture faire aujourd'hui de ces commentaires? Comment prendre en compte ces annotations en termes de métadonnées? Quelle analyse au regard des évolutions de la discipline?

Les matériaux accumulés par les géographes peuvent refléter un regard distancié, exotisant, c'est-à-dire qui définit une altérité, le point de vue du géographe issu d'un pays du « Nord » qui porte un regard sur « les Suds », qui s'approprie une légitimité scientifique dans la mise à distance de sociétés considérées comme exotiques.

Par exemple, une catégorie analytique comme celle de «tradition», utilisée par certains géographes et reprise dans certaines métadonnées, a fait l'objet de multiples critiques et analyses, en anthropologie notamment. La notion de «tradition» fait référence à une transmission lointaine mais non définie. Qualifier un objet culturel de «traditionnel » l'oppose à l'idée de «modernité ». Il représente un temps passé, qui peut être perçu comme dévalorisé. D’autres catégories peuvent susciter le même type de réflexion. Par exemple, «habitat spontané » est une expression qui a pu être utilisée pour qualifier un habitat

\footnotetext{
${ }^{1}$ http:/ / ethiquedroit.hypotheses.org
} 
improvisé, dans un contexte de précarité. Ce terme induit une notion d'illégalité impossible à vérifier sur une photographie. La notion de «logement insalubre » peut induire un jugement de valeur, etc.

Pour le documentaliste ou l'archiviste, il s'agit de prendre en compte ce contexte, de le comprendre et de ne pas forcément réutiliser les mêmes catégories. En tant que documentalistes, archivistes, producteurs de métadonnées de description, ici concernant des images, nous avons donc la responsabilité d'une réflexion sur la façon dont ces métadonnées construisent et traduisent de manière sélective des ensembles et des frontières culturelles. Même si toutes les catégories analytiques utilisées par les géographes ne renvoient pas nécessairement à des stéréotypes, la façon dont des objets ou sujets sont qualifiés au sein d'une catégorie de description est indissociable des points de vue théoriques qui ont motivé ces qualifications.

Quelle solution ? Il s'agit justement de contextualiser, de garder et donner à voir l'historicité d'une information ${ }^{1}$. Si une catégorie analytique reflète un stéréotype lié à un mode de pensée, dans tous les cas, puisqu'elle s'inscrit dans une histoire, il faudrait le préciser. Cela passe également par un travail sur les ontologies utilisées aujourd'hui : le vocabulaire, les concepts ancrés, eux aussi, dans une histoire disciplinaire contemporaine.

\section{Restitution}

Les travaux d'archivage menés dans le cadre du centre d'IST «Regards » et d'ImaGEO s'inscrivent dans une démarche de type «science ouverte », c'est-àdire en faveur d'un accès ouvert aux données, sinon aux métadonnées produites, afin d'assurer un meilleur rayonnement de la recherche, une réutilisation mais aussi une restitution possible.

Dans ce cadre et par rapport au contexte de production des données que nous venons d'évoquer, le retour de celles-ci (des photographies mais également des cartes) dans les pays dont elles sont issues représente un enjeu important.

\footnotetext{
1 À ce sujet, voir par ailleurs la présentation de Laure Carbonnel, p. 13.
} 
Plus globalement, cet enjeu autour du patrimoine scientifique et du patrimoine culturel en général a fait l'objet de nombreuses publications, dans le contexte de revendications communautaires pour un retour des archives patrimoniales.

De quelle manière se traduit ici ce "retour »? Il s'agit d'ouvrir l'accès aux données, ou a minima aux métadonnées, pour un partage des connaissances. Cet accès ouvert concerne les données de plus de soixante-dix ans pour les cartes et un accès illimité à toutes les collections photographiques de terrain des chercheurs. La première forme de restitution est donc la mise en ligne.

Une autre forme de restitution se traduit par le retour des données elles-mêmes sur leur terrain d'origine. Un exemple emblématique est celui d'une exposition de cartes de Madagascar dans ce pays. Nous avons en effet participé, avec un autre partenaire d'ImaGEO, la cartothèque de l'université Bordeaux Montaigne, à la réalisation d'une exposition intitulée "Madagascar - Cartographie d'hier et cartographie d'aujourd'hui - Diego et sa baie vues d'en haut». Cette exposition présentait de nombreuses reproductions de cartes issues de nos fonds patrimoniaux de la fin du XIX ${ }^{\mathrm{e}}$ siècle à nos jours sur la ville de Diego Suarez (Antsiranana en malgache). Le commissaire scientifique était un chercheur membre de l'UMR Passages à Bordeaux, Xavier Amelot. Elle a été mise en œuvre grâce à un partenariat entre l'université Bordeaux Montaigne et l'université Nord Madagascar d'Antsiranana. Une association locale pour la préservation du patrimoine historique et environnemental de la région Diana était également impliquée ${ }^{1}$. L'exposition s'est tenue à l'Alliance française du 7 au 18 novembre 2016, dans un ancien marché couvert du centre historique de Diego Suarez. Elle s'inscrivait dans le cadre de l'année internationale de la cartographie. Dans un pays où l'information scientifique est difficilement accessible et où la dernière production de cartes topographiques date de plus de cinquante ans, elle s'adressait à tous les publics, en particulier scolaire. Le rôle des personnes impliquées dans cette exposition, chercheurs, service de reprographie, documentalistes et bibliothécaires a été de fournir, grâce aux travaux de numérisation en haute définition, des documents de très bonne qualité, sans transport des cartes, pour continuer à en assurer une bonne conservation.

Cette exposition a permis de s'interroger de façon critique sur l'histoire de la cartographie de l'île et sur les changements, les évolutions des représentations territoriales de l'île (entre autres par rapport aux questions de déforestation et

\footnotetext{
${ }^{1}$ Association Ambre, Antsiranana, Madagascar.
} 
aux choix cartographiques qui ont été élaborés pour la représenter). Elle a permis d'actualiser les connaissances sur cet espace géographique ${ }^{1}$.

\title{
Conclusion
}

Nous avons abordé deux exemples de responsabilités.

Il a été question de responsabilité au sens d'une réflexion que peuvent mener documentalistes et archivistes sur les modalités de traitement documentaire et archivistique effectué, par rapport aux concepts descriptifs utilisés, par rapport aux mots-clefs d'une indexation de ces archives, et il a été question de responsabilité également par rapport à l'utilisation de ces archives.

La première fait l'objet de recherches épistémologiques, elle évolue en même temps que la discipline géographique et les sciences humaines et sociales voient leurs modes de pensée se transformer aussi. La seconde est complexe, elle nécessite des négociations, des échanges avec des partenaires des Suds. C'est ce que souhaite développer en particulier ImaGEO en s'ouvrant à ces partenariats et en même temps à la société civile.

\author{
Caroline ABELA \\ Ingénieure CNRS \\ Centre IST Regards - UMR Passages \\ caroline.abela@cnrs.fr
}

\footnotetext{
1 Voir à ce sujet le billet du carnet d'ImaGEO «Exposition : "Madagascar - Cartographie d'hier et cartographie d'aujourd'hui”" » (30/03/2016) : https://imageo.hypotheses.org/49
} 